\title{
Upper Bounds on MIMO Channel Capacity with Channel Frobenius Norm Constraints
}

\author{
Zukang Shen, Jeffrey G. Andrews, and Brian L. Evans \\ Wireless Networking and Communications Group \\ Department of Electrical and Computer Engineering \\ The University of Texas at Austin, Austin, Texas 78712 \\ Email: $\{$ shen, jandrews, bevans\}@ece.utexas.edu
}

\begin{abstract}
The motivation of this paper is to find the class of channels that provides the largest capacity with both the transmit power constraint and channel Frobenius norm constraints. We study both the point-to-point case and the broadcast case. For point-to-point MIMO channels, the optimal channels must have $T^{*}$ equal singular values, where $T^{*}$ is dependent on the available transmit power and the channel Frobenius norm. This result agrees with the previous work by Chiurtu et al. For multiuser broadcast channels, we obtain an upper bound on the sum capacity. We also show that the bound is asymptotically tight for high SNR when all user channels have the same Frobenius norm constraint and $N_{t} \geq K N_{r}$, where $N_{t}$ and $N_{r}$ are the numbers of transmit and receive antennas and $K$ is the number of users.
\end{abstract}

\section{INTRODUCTION}

Multiple-input-multiple-output (MIMO) systems have drawn a lot of attention in the last decade. The spatial dimension, in addition to the time and frequency dimensions, can be exploited with multiple antennas at the transmitter and receiver. The fundamental information theoretic results on MIMO channel capacity, e.g. [1]-[6] just to name a few, are important because they not only show the performance limits, but also shed light on how to achieve the capacity, which helps to design MIMO systems.

The Gaussian MIMO channel capacity problem can be generally formulated into an optimization problem. Given a fixed channel or a random channel with certain probability distributions, the objective is to optimize the input covariance matrix under a power constraint so that the channel capacity is achieved. For example, for a fixed channel $\mathbf{H}$, the point-topoint MIMO channel capacity can be formulated as

$$
\begin{gathered}
\underset{\mathbf{Q}}{\max } \log \left|\mathbf{I}+\frac{1}{\sigma^{2}} \mathbf{H} \mathbf{Q} \mathbf{H}^{\dagger}\right| \\
\text { subject to } \operatorname{tr}(\mathbf{Q}) \leq P
\end{gathered}
$$

where $\mathbf{Q}$ is the input covariance matrix and the only constraint in (1) is the power constraint, i.e. $\operatorname{tr}(\mathbf{Q}) \leq P$. While the MIMO channel capacity is a function of the available transmit power, it is also dependent on the channel characteristics, such as the channel power. In [7], Sayeed et al. studied the optimal

This work was supported by a gift from Texas Instruments and an equipment donation from Intel.
MIMO channel capacity scaling law under different channel power scaling laws with respect to the number of antennas. While [7] focused on the optimal capacity scaling law, in this paper, we study the maximum channel capacity with both transmit power and channel power (represented by the channel Frobenius norm) constraints.

The term maximum capacity in this paper refers to the maximum capacity over all possible channels satisfying the norm constraint, while capacity is defined to be the maximum mutual information over the transmit signal covariance matrix for a fixed channel. The motivation for optimizing the channel jointly with the input covariance matrix under the transmit power and channel power constraints are: 1) for the class of power-constrained channels, an upper bound on the MIMO channel capacity can be found; and 2) the characteristics of the channels providing the maximum capacity can be obtained, which may be used to direct the adaptive antenna array configuration if possible.

We study both single user point-to-point MIMO systems and multiuser broadcast channels. For the point-to-point case, Chiurtu et al. in [8] showed that the class of channels that provides maximum capacity must have equal singular values, and the optimal transmit signal covariance matrix also assigns equal power to those non-zero eigenmodes. While Chiurtu et al. obtained the solution with Lagrange multipliers in [8], we study the problem using iterative water-filling. For the multiuser broadcast channel case, we obtain an upper bound on the largest sum capacity for the channels bounded by Frobenius norm. We also show that the bound is asymptotically tight for high SNR when $N_{t} \geq K N_{r}$ and all user channels have the same Frobenius norm constraint.

\section{System Model AND Problem Formulation}

\section{A. Single User: Point-to-point Case}

The symbolwise discrete-time input-output relationship of a narrowband point-to-point MIMO system can be written as

$$
\mathbf{y}=\mathbf{H x}+\mathbf{v}
$$

where $\mathbf{H}$ is the $N_{r} \times N_{t}$ channel matrix; $\mathbf{x}$ and $\mathbf{y}$ are the transmitted and received symbol vector, respectively; $\mathbf{v}$ is the additive white Gaussian noise vector, with $E\left[\mathbf{v v}^{\dagger}\right]=\sigma^{2} \mathbf{I}$, and $(\cdot)^{\dagger}$ denotes matrix complex conjugate transpose. Without loss of generality, we assume $\sigma^{2}=1$. 
In this paper, we consider the MIMO channel capacity with both total transmit power constraint and channel Frobenius norm constraint. The motivation is to find the bounds on the MIMO channel capacity with a channel Frobenius constraint, i.e. $\|\mathbf{H}\|_{F}^{2} \leq W$, to identify the channels maximize capacity. For a point-to-point MIMO system, we have the following problem formulation.

$$
\begin{array}{r}
\max _{\mathbf{H}} \max _{\mathbf{Q}} \log \left|\mathbf{I}+\mathbf{H Q H}^{\dagger}\right| \\
\text { subject to }: \operatorname{tr}(\mathbf{Q}) \leq P \\
\|\mathbf{H}\|_{F}^{2} \leq W .
\end{array}
$$

\section{B. Multiple Users: Broadcast Case}

For a broadcast multiuser MIMO system, the received signal for user $k$ can be expressed as

$$
\mathbf{y}_{k}=\mathbf{H}_{k} \sum_{i=1}^{K} \mathbf{x}_{i}+\mathbf{v}_{k}
$$

where $\mathbf{x}_{i}$ is the user $i$ 's transmitted signal; $\mathbf{H}_{k}$ of size $N_{r} \times N_{t}$ denotes the channel for user $k ; \mathbf{y}_{k}$ is the received signal for user $k ; \mathbf{v}_{k}$ is the additive white Gaussian noise at receiver $k$; and $K$ is the total number of users. Furthermore, in this paper, we assume all users are equipped with an equal number of receiver antennas $N_{r}$ and the transmitter has $N_{t} \geq N_{r}$ transmit antennas.

It has been shown that Dirty Paper Coding (DPC) [10] achieves the sum capacity in a Gaussian broadcast MIMO channel [13]. And the duality results in [13] show that for a fixed set of channels $\left\{\mathbf{H}_{k}\right\}_{k=1}^{K}$, the sum capacity of a Gaussian broadcast MIMO channel can found by solving the following problem:

$$
\begin{array}{r}
\max _{\left\{\mathbf{Q}_{k}\right\}} \log \left|\mathbf{I}+\sum_{k=1}^{K} \mathbf{H}_{k}^{\dagger} \mathbf{Q}_{k} \mathbf{H}_{k}\right| \\
\text { subject to } \sum_{k=1}^{K} \operatorname{Tr}\left(\mathbf{Q}_{k}\right) \leq P .
\end{array}
$$

Similar to the point-to-point case, with a set of channel Frobenius norm constraints, we have the following problem formulation for a Gaussian broadcast channel:

$$
\begin{array}{ll} 
& \max _{\left\{\mathbf{H}_{k}\right\}} \max _{\left\{\mathbf{Q}_{k}\right\}} \log \left|\mathbf{I}+\sum_{k=1}^{K} \mathbf{H}_{k}^{\dagger} \mathbf{Q}_{k} \mathbf{H}_{k}\right| \\
\text { subject to } & \sum_{k=1}^{K} \operatorname{Tr}\left(\mathbf{Q}_{k}\right) \leq P \\
& \left\|\mathbf{H}_{k}\right\|_{F}^{2} \leq W_{k} \quad \text { for } k=1,2, \cdots, K .
\end{array}
$$

Remarks: The emphasis of this paper is to find what kind of channels provide the maximum MIMO channel capacity with a channel norm constraint. It is assumed that the transmitter knows the channel perfectly in order to optimize its signal covariance matrix.

\section{Point-To-POINT CASE}

Let $N=\max \left\{N_{t}, N_{r}\right\}$ and $M=\min \left\{N_{t}, N_{r}\right\}$, and let the singular value decomposition (SVD) of $\mathbf{H}^{\dagger} \mathbf{H}$ be $\mathbf{H}^{\dagger} \mathbf{H}=$ $\mathbf{U D U}^{\dagger}$, where $\mathbf{D}$ is a diagonal matrix whose $M$ non-zero elements are denoted as $\left\{\lambda_{i}\right\}_{i=1}^{M}$; and $\mathbf{U}$ is a unitary matrix. Notice that

$$
\sum_{i=1}^{M} \lambda_{i}=\|\mathbf{H}\|_{F}^{2}
$$

and

$$
\log \left|\mathbf{I}+\mathbf{H Q H}^{\dagger}\right|=\sum_{i=1}^{M} \log \left(1+S_{i} \lambda_{i}\right)
$$

where $S_{i}$ is the power allocated to the $i$ th non-zero eigenmode, the original problem in (3) can be reformulated as

$$
\begin{aligned}
& \max _{\boldsymbol{\Lambda}} \max _{\mathbf{S}} \sum_{i=1}^{M} \log \left(1+S_{i} \lambda_{i}\right) \\
& \text { subject to }: \sum_{i=1}^{M} S_{i} \leq P \\
& \sum_{i=1}^{M} \lambda_{i} \leq W
\end{aligned}
$$

where $\mathbf{S}=\left\{\begin{array}{llll}S_{1} & S_{2} & \cdots & S_{M}\end{array}\right\}$ and $\boldsymbol{\Lambda}=\left\{\begin{array}{llll}\lambda_{1} & \lambda_{2} & \cdots & \lambda_{M}\end{array}\right\}$.

Notice that the objective function in (9) is symmetric with $\mathbf{S}$ and $\boldsymbol{\Lambda}$. For a given $\boldsymbol{\Lambda}$, the optimal $S_{i}=\Gamma_{s}-\frac{1}{\lambda_{i}}$ is obtained by water-filling, where $\Gamma_{s}$ is the water level which satisfies $\sum_{i=1}^{M} S_{i}=P$. For a given $\mathbf{S}$, the optimal $\lambda_{i}=\Gamma_{\lambda}-\frac{1}{S_{i}}$ and $\sum_{i=1}^{M} \lambda_{i}=W$. Hence iterative water-filling can be used between $\mathbf{S}$ and $\boldsymbol{\Lambda}$, i.e.

1) starting from an initial value $\mathbf{S}^{*}$, maximize the objective function in (9) over $\boldsymbol{\Lambda}$. And let $\boldsymbol{\Lambda}^{*}=$ $\arg \max _{\Lambda} \sum_{i=1}^{M} \log \left(1+S_{i}^{*} \lambda_{i}\right)$.

2) For the $\Lambda^{*}$, maximize the objective function in (9) over S. Let $\mathbf{S}^{*}=\arg \max _{\mathbf{S}} \sum_{i=1}^{M} \log \left(1+S_{i} \lambda_{i}^{*}\right)$.

3) Repeat step 1 and 2 until an equilibrium is reached.

Based on the above iterative water-filling procedure, we have the following two Lemmas:

Lemma 1: At each equilibrium point, the following equations hold

$$
\begin{aligned}
& S_{i}^{*}=\Gamma_{s}-\frac{1}{\lambda_{i}^{*}} \text { for } i=1,2, \cdots, T \\
& \lambda_{i}^{*}=\Gamma_{\lambda}-\frac{1}{S_{i}^{*}} \text { for } i=1,2, \cdots, T \\
& \sum_{i=1}^{T} S_{i}^{*}=P \\
& \sum_{i=1}^{T} \lambda_{i}^{*}=W
\end{aligned}
$$

for some $1 \leq T \leq M$.

Proof: Equations (10) and (11) are easy to justify because at each equilibrium point, $\left\{S_{i}^{*}\right\}_{i=1}^{T}$ are the water-filling results for $\left\{\lambda_{i}^{*}\right\}_{i=1}^{T}$. Otherwise, the iterative water-filling procedure 
can be continued until an equilibrium point is reached. Equations (12) and (13) are true because the transmitter is supposed to use as much power as possible and the channel shall also provide maximal energy. Hence, the power and channel norm constraints are binding.

Lemma 2: The global maxima must be one of these equilibrium points.

Proof: This result is straightforward because the objective function, i.e. $\sum_{i=1}^{M} \log \left(1+S_{i} \lambda_{i}\right)$ is not decreasing as the iterative water-filling procedure progresses. Suppose the global maxima does not satisfy the conditions in Lemma 1, then performing the iterative water-filling algorithm can further improve the objective function.

Theorem 1: For a fixed channel $\mathbf{H}$ with channel norm constraint $\|\mathbf{H}\|_{F}^{2} \leq W$ and transmit power constraint $\operatorname{Tr}(\mathbf{Q}) \leq$ $P$, the maximum possible channel capacity is

$$
\max _{\mathbf{Q}, \mathbf{H}} \log \left|\mathbf{I}+\mathbf{H Q} \mathbf{H}^{\dagger}\right|=\max _{T: T=1,2, \cdots, M} T \log \left(1+\frac{P W}{T^{2}}\right) .
$$

Proof: First, we will find the equilibrium points from Lemma 1.

Multiply (10) with $\Lambda_{i}^{*}$ and multiply (11) with $S_{i}^{*}$ to obtain

$$
\begin{aligned}
& S_{i}^{*} \lambda_{i}^{*}=\Gamma_{s} \lambda_{i}^{*}-1 \\
& \lambda_{i}^{*} S_{i}^{*}=\Gamma_{\lambda} S_{i}^{*}-1
\end{aligned}
$$

Hence

$$
S_{i}^{*}=\frac{\Gamma_{s}}{\Gamma_{\lambda}} \lambda_{i}^{*}
$$

for $i=1,2, \cdots, T$. Substituting (17) into (11) results in

$$
\lambda_{i}^{*}=\Gamma_{\lambda}-\frac{\Gamma_{\lambda}}{\Gamma_{s}} \frac{1}{\lambda_{i}^{*}}
$$

for $i=1,2, \cdots, T$. Notice that from (18), $\lambda_{i}^{*}$ only depends on $\Gamma_{\lambda}$ and $\Gamma_{s}$, which is the same for all $i=1,2, \cdots, T$. Although $\lambda_{i}^{*}$ may be one of the two possible solutions to (18), the Lemma in [8] proves that in order to maximize (9), we must have

$$
\lambda_{i}^{*}=\lambda_{j}^{*}
$$

and

$$
S_{i}^{*}=S_{j}^{*}
$$

for $1 \leq i, j \leq T$. Combined with (12) and (13), we obtain

$$
S_{i}^{*}=\frac{P}{T}
$$

and

$$
\lambda_{i}^{*}=\frac{W}{T}
$$

for $i=1,2, \cdots, T$. Also it is easy to see that

$$
\frac{\Gamma_{s}}{\Gamma_{\lambda}}=\frac{P}{W} .
$$

Notice that there are $M$ (for $1 \leq T \leq M$ ) candidate equilibrium points for the global maxima. From Lemma 2, the global maximum of the original problem in (3) can be obtained by finding the maximum among those $M$ equilibrium points, i.e.

$$
\max _{\mathbf{Q}, \mathbf{H}} \log \left|\mathbf{I}+\mathbf{H} \mathbf{Q} \mathbf{H}^{\dagger}\right|=\max _{T: T=1,2, \cdots, M} T \log \left(1+\frac{P W}{T^{2}}\right)
$$

which is straightforward to evaluate.

Let $T^{*}=\arg \max _{T: T=1,2, \cdots, M} T \log \left(1+\frac{P W}{T^{2}}\right)$. The interpretation of the above results is that among all possible channel $\mathbf{H}$ which is limited by the Frobenius norm constraint, i.e. $\| \mathbf{H}||_{F}^{2} \leq W$, the class of $\mathbf{H}$ maximizing the channel capacity has $T^{*}$ equal singular values of $\sqrt{W / T^{*}}$. Notice that the product of $P$ and $W$ completely decides $T^{*}$, leading to the following observation.

Lemma 3: For sufficiently small $P W, T^{*}=1$, and for sufficiently large $P W, T^{*}=M$.

This is reasonable because when $P W$ is sufficiently small, those channels providing the largest capacity shall focus both the channel energy and transmit power to one eigenmode. This is somehow related to the optimality of beamforming in low SNR regime. When $P W$ is sufficiently large, then $T^{*}=M$, meaning all possible spatial dimensions shall be utilized.

\section{BROADCAST CASE}

For a multi-user broadcast channel, the optimization problem in (6) is difficult to solve. Even for a set of fixed user channels, the boradcast sum capacity in (5) is a difficult optimization problem. Several algorithms have been proposed, e.g. the iterative water-filling algorithm by Jindal, et al. in [16]. In this section, we show an upper bound for the problem in (6) in following theorem.

Theorem 2: For multiuser broadcast channels with individual channel norm constraints $\left\|\mathbf{H}_{k}\right\|_{F}^{2} \leq W_{k}$ for $k=1,2, \cdots, K$, and a total transmit power constraint $\sum_{k=1}^{K} \operatorname{Tr}\left(\mathbf{Q}_{k}\right) \leq P$, the maximum sum capacity is upper bounded by

$$
\begin{array}{r}
\max _{\left\{\mathbf{H}_{k}\right\}:\left\|\mathbf{H}_{k}\right\|_{F}^{2} \leq W_{i}} \max _{\left\{\mathbf{Q}_{k}\right\}: \sum_{k=1}^{K} \operatorname{Tr}\left(\mathbf{Q}_{k}\right) \leq P} \log \left|\mathbf{I}+\sum_{k=1}^{K} \mathbf{H}_{k}^{\dagger} \mathbf{Q}_{k} \mathbf{H}_{k}\right| \\
\leq \max _{T: T=1,2, \cdots, M_{c}} T \log \left(1+\frac{P \sum_{k=1}^{K} W_{k}}{T^{2}}\right)
\end{array}
$$

where $M_{c}=\min \left\{N_{t}, K N_{r}\right\}$.

Proof: Notice that if we denote $\mathbf{H}_{c}=\left[\begin{array}{llll}\mathbf{H}_{1}^{\dagger} & \mathbf{H}_{2}^{\dagger} & \cdots & \mathbf{H}_{K}^{\dagger}\end{array}\right]^{\dagger}$, then

$$
\begin{array}{r}
\max _{\left\{\mathbf{Q}_{k}\right\}: \sum_{k=1}^{K} \operatorname{Tr}\left(\mathbf{Q}_{k}\right) \leq P} \log \left|\mathbf{I}+\sum_{k=1}^{K} \mathbf{H}_{k}^{\dagger} \mathbf{Q}_{k} \mathbf{H}_{k}\right| \\
\leq \max _{\mathbf{Q}: \operatorname{Tr}(\mathbf{Q}) \leq P} \log \left|\mathbf{I}+\mathbf{H}_{c} \mathbf{Q} \mathbf{H}_{c}^{\dagger}\right| .
\end{array}
$$

This is true because the right hand side (RHS) of (26) is the capacity of a point-to-point channel $\mathbf{H}_{c}$, which represents the scenario in which users may cooperate in the broadcast channel. 
Since (26) holds true for any channel $\left\{\mathbf{H}_{k}\right\}_{k=1}^{K}$, and notice that $\left\|\mathbf{H}_{c}\right\|_{F}^{2}=\sum_{k=1}^{K}\left\|\mathbf{H}_{k}\right\|_{F}^{2}$, we have

$$
\begin{aligned}
& \max _{\left\{\mathbf{H}_{k}\right\}:\left\|\mathbf{H}_{k}\right\| \|_{F}^{2} \leq W_{i}} \max _{\left\{\mathbf{Q}_{k}\right\}: \sum_{k=1}^{K} \operatorname{Tr}\left(\mathbf{Q}_{k}\right) \leq P} \log \left|\mathbf{I}+\sum_{k=1}^{K} \mathbf{H}_{k}^{\dagger} \mathbf{Q}_{k} \mathbf{H}_{k}\right| \\
& \leq \max _{\mathbf{H}_{c}:\left\|\mathbf{H}_{c}\right\|_{F}^{2} \leq \sum_{k=1}^{K} W_{k} \mathbf{Q}: \operatorname{Tr}(\mathbf{Q}) \leq P} \log \left|\mathbf{I}+\mathbf{H}_{c} \mathbf{Q} \mathbf{H}_{c}^{\dagger}\right|
\end{aligned}
$$

Let $M_{c}=\min \left\{N_{t}, K N_{r}\right\}$. From Theorem 1, the solution to the RHS of (27) can be easily obtained as

$$
\begin{array}{r}
\max _{\mathbf{H}_{c}:\left\|\mathbf{H}_{c}\right\|_{F}^{2} \leq \sum_{k=1}^{K} W_{k} \mathbf{Q}: \operatorname{Tr}(\mathbf{Q}) \leq P} \log \left|\mathbf{I}+\mathbf{H}_{c} \mathbf{Q} \mathbf{H}_{c}^{\dagger}\right| \\
=\max _{T: T=1,2, \cdots, M_{c}} T \log \left(1+\frac{P \sum_{k=1}^{K} W_{k}}{T^{2}}\right)
\end{array}
$$

Hence combining (27) and (28) completes the proof.

Theorem 2 only gives an upper bound on the maximum sum capacity with channel norm constraints. In the following, we show a special case where the upper bound in Theorem 2 is actually tight.

Before stating the Lemma, we first define some notation. Let $T^{*}$ be the maxima of the RHS of (28), i.e.

$$
T^{*}=\arg \max _{T: T=1,2, \cdots, M_{c}} T \log \left(1+\frac{P \sum_{k=1}^{K} W_{k}}{T^{2}}\right) .
$$

Let the SVD of $\mathbf{H}_{k}=\mathbf{U}_{k} \mathbf{D}_{k} \mathbf{V}_{k}^{\dagger}$, where $\mathbf{U}_{k}$ is a unitary matrix of size $N_{r} \times N_{r} ; \mathbf{V}_{k}$ is a unitary matrix of size $N_{t} \times N_{r}$; and $\mathbf{D}_{k}$ is a diagonal matrix of size $N_{r} \times N_{r}$ the diagonal elements denoted as $\left\{\lambda_{k, i}\right\}_{i=1}^{N_{r}}$.

Lemma 4: When $T^{*}=K \bar{N} \leq N_{t}$ for some integer $1 \leq$ $\bar{N} \leq N_{r}$ and $W_{1}=W_{2}=\cdots=W_{K}=W$, the bound in (25) is tight.

Proof: With the conditions given in Lemma 4, the bound in (25) becomes

$$
\log \left(1+\frac{P K W}{(K \bar{N})^{2}}\right)^{K \bar{N}}=\log \left(1+\frac{P W}{K \bar{N}^{2}}\right)^{K N_{T}}
$$

In the following, we construct a set of user channels $\left\{\mathbf{H}_{k}\right\}_{k=1}^{K}$ such that the bound in (29) is achieved.

- Let $\mathbf{V}_{1} \perp \mathbf{V}_{2} \perp \cdots \perp \mathbf{V}_{k}$, which means all user channels

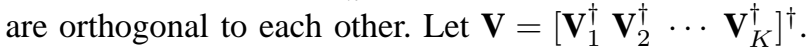

- Let $\mathbf{D}_{k}=\operatorname{diag}\left\{\sqrt{\frac{W}{\bar{N}}} \sqrt{\frac{W}{\bar{N}}} \cdots \sqrt{\frac{W}{\bar{N}}} 0 \cdots 0\right\}$ for $k=$ $1,2, \cdots, K$.

- Let $\mathbf{E}=\operatorname{diag}\left\{\frac{P}{K \bar{N}} \frac{P}{K \bar{N}} \cdots \frac{P}{K \bar{N}} 0 \cdots 0\right\}$. Let $\mathbf{Q}_{k}=$ $\mathbf{U}_{k} \mathbf{E} \mathbf{U}_{k}^{\dagger}$ for $k=1,2, \cdots, K$.
Then

$$
\begin{aligned}
\left|\mathbf{I}+\sum_{k=1}^{K} \mathbf{H}_{k}^{\dagger} \mathbf{Q}_{k} \mathbf{H}_{k}\right| & =\left|\mathbf{I}+\sum_{k=1}^{K} \mathbf{V}_{k} \mathbf{D}_{k} \mathbf{U}_{k}^{\dagger} \mathbf{U}_{k} \mathbf{E} \mathbf{U}_{k}^{\dagger} \mathbf{U}_{k} \mathbf{D}_{k} \mathbf{V}_{k}^{\dagger}\right| \\
& =\left|\mathbf{I}+\sum_{k=1}^{K} \mathbf{V}_{k} \mathbf{D}_{k}^{2} \mathbf{E} \mathbf{V}_{k}^{\dagger}\right| \\
& \stackrel{(a)}{=}\left|\mathbf{I}+\mathbf{V D V} \mathbf{V}^{\dagger}\right| \\
& =|\mathbf{I}+\mathbf{D}|
\end{aligned}
$$

where $\mathbf{D}$ is a diagonal matrix of size $K N_{r} \times K N_{r}$, It has $K \bar{N}$ non-zero diagonal element and the $j$ th element $d_{j}$ is

$$
d_{j}=\frac{P}{K \bar{N}} \frac{W}{\bar{N}}
$$

for all $j=1,2, \cdots, K \bar{N}$. And equality (a) holds because $\mathbf{V}_{1} \perp \mathbf{V}_{2} \perp \cdots \perp \mathbf{V}_{k}$ and $\mathbf{D}_{k}^{2} \mathbf{E}$ is diagonal for all $k=$ $1,2, \cdots, K$.

With (31), we have

$$
\begin{aligned}
\log \left|\mathbf{I}+\sum_{k=1}^{K} \mathbf{H}_{k}^{\dagger} \mathbf{Q}_{k} \mathbf{H}_{k}\right| & =\prod_{j=1}^{K \bar{N}} \log \left(1+d_{j}\right) \\
& =K \bar{N} \log \left(1+\frac{P W}{K \bar{N}^{2}}\right)
\end{aligned}
$$

with equals (29). Hence Lemma 3 is proved.

Lemma 5: When $K N_{r} \leq N_{t}$ and $W_{1}=W_{2}=\cdots=$ $W_{K}=W$, the bound in (25) is asymptotically tight for high SNR.

Proof: For high SNR, we have $T^{*}=\min \left\{N_{t}, K N_{r}\right\}=$ $K N_{r}$. This is true because for large enough SNR, the transmitter needs to utilize as many eigenmodes as possible. Hence Lemma 5 is a special case of Lemma 4 where $\bar{N}=N_{r}$.

Remarks: The results in Lemma 4 and Lemma 5 are not surprising because when the user channels are mutually orthogonal, the sum capacity of the broadcast channel is the same as the cooperative channel with the same total transmit power constraint.

\section{Numerical RESUlts}

In this section, we provide a few numerical results on the maximum MIMO channel capacity with both transmit power and channel norm constraints.

For a point-to-point MIMO channel, since the optimal number of non-zero eigenmodes $T^{*}$ is determined by the product of $P$ and $W$, we define SNR $=P W$ in this paper. Fig. 1 shows the largest possible MIMO channel capacity vs. SNR for different $M$, which is the minimum of the number of transmit and receive antennas. For low SNR, the capacity is the same regardless of the value of $M$. This is true since under low SNR, the optimal transmit strategy is to allocate all power to the largest eigenmode. And those channels providing the most capacity shall focus all its energy to one eigenmode. As SNR increases, more eigenmodes shall be utilized. And Fig. 2 shows the optimal number of eigenmodes vs. SNR. Fig. 3 shows the largest capacity vs. $M$ for different SNR. It 


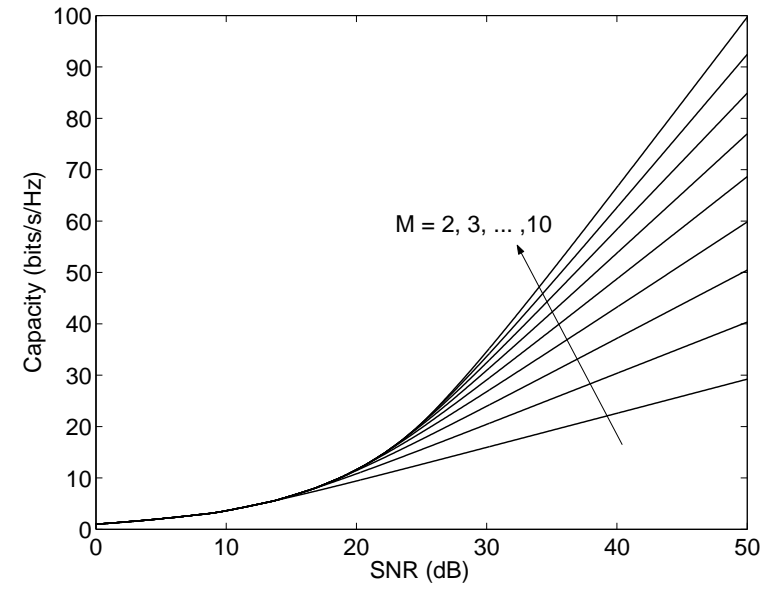

Fig. 1. Point-to-point MIMO channels. Maximum capacity vs. SNR, which is defined as $\mathrm{SNR} \triangleq P W . M=\min \left\{N_{t}, N_{r}\right\}$.

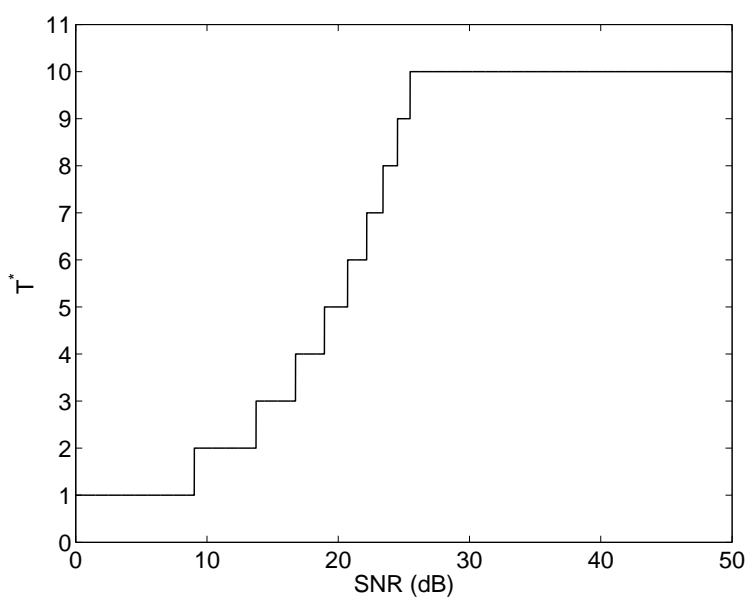

Fig. 2. Point-to-point MIMO channels. Optimal number of eigenmodes $\left(T^{*}\right)$ vs. SNR, which is defined as $\mathrm{SNR} \triangleq P W . M=\min \left\{N_{t}, N_{r}\right\}=10$.

is simply a different view of Fig. 1. From Fig. 3, we confirmed that for low SNR, the largest capacity is the same for all $M$.

\section{CONCLUSION}

In this paper, the MIMO channel capacity with channel Frobenius norm constraints is studied. For a point-to-point MIMO channel, the optimal channel that provides the largest capacity must have $T^{*}$ equal eigenmodes, where $T^{*}$ is dependent on the total transmit power constraint $P$ and the channel norm constraint $W$. For a multiuser broadcast channel, we obtain an upper bound on the largest sum capacity when the user channels have Frobenius norm constraints. It is also shown that the bound is asymptotically tight for high SNR when $N_{t} \geq K N_{r}$ and all user channels have the same Frobenius norm constraint.

\section{REFERENCES}

[1] I. E. Telatar, "Capacity of Multi-Antenna Gaussian channels," European Transactions on Telecommunications, vol. 10, no. 6, pp. 585-595, Nov./Dec. 1999.

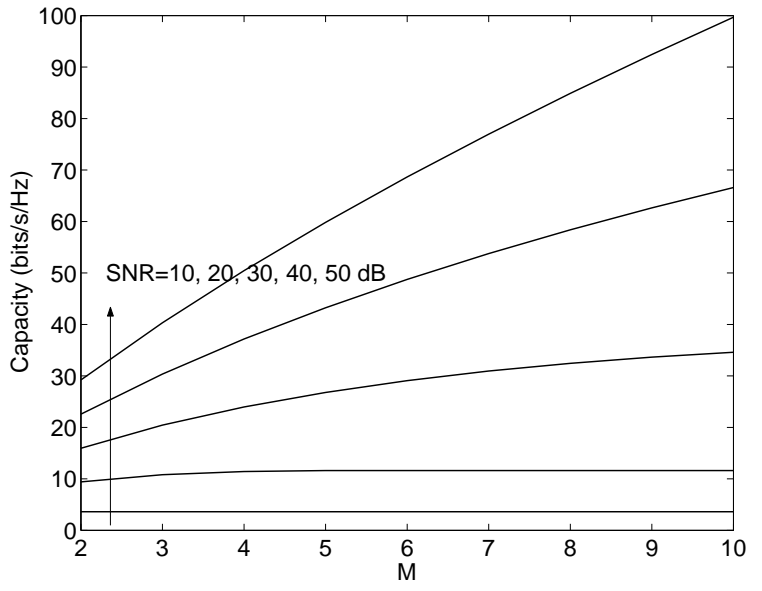

Fig. 3. Point-to-point MIMO channels. Maximal capacity vs. M, which is defined as $M=\min \left\{N_{t}, N_{r}\right\}$. And $\mathrm{SNR} \triangleq P W$.

[2] A. Goldsmith, S. A. Jafar, N. Jindal, and S. Vishwanath, "Capacity Limits of MIMO Channels," IEEE Journal on Selected Area in Communications, vol. 21, no. 5, p. 684-702, Jun. 2003.

[3] S. K. Jayaweera and H. V. Poor, "Capacity of Multiple-Antenna Systems with Both Receiver and Transmitter Channel State Information," IEEE Transactions on Information Theory, vol. 49, no. 10, pp. 2697-2709, Oct. 2003.

[4] E. Biglieri, G. Caire, and G. Taricco, "Limiting Performance of BlockFading Channels with Multiple Antennas," IEEE Transactions on Information Theory, vol. 47, no. 4, pp. 1273-1289, May 2001.

[5] A. S. Y. Poon, D. N. C. Tse, and R. W. Brodersen, "An Adaptive Multiantenna Transceiver for Slowly Flat Fading Channels," IEEE Transactions on Communications, vol. 51, no. 11, pp. 1820-1827, Nov. 2003.

[6] Z. Shen, R. W. Heath. Jr., J. G. Andrews, and B. L. Evans, "Comparison of Space-Time Water-filling and Spatial Water-filling for MIMO Fading Channels," in Proc. IEEE Global Communications Conference., vol. 1, pp. 431-435, Dec. 2004

[7] A. Sayeed, V. Raghavan, and J. Kotecha, "Capacity of Space-Time Wireless Channels: A Physical Perspective," in IEEE Information Theory Workshop, Oct. 2004.

[8] N. Chiurtu and B. Rimoldi, "Varying the Antenna Locations to Optimize the Capacity of Multi-Antenna Gaussian Channels," in Proc. IEEE International Conference on Acoustics, Speech, and Signal Processing, vol. 5, pp. 3121-3123, Jun. 2000.

[9] N. Chiurtu, B. Rimoldi, and E. Telatar, "On the Capacity of MultiAntenna Gaussian Channels," in Proc. IEEE International Symposium on Information Theory, pp. 53, Jun. 2001.

[10] M. Costa, "Writing on Dirty Paper," IEEE Transactions on Information Theory, vol. 29, no. 3, pp. 439-441, May 1983.

[11] G. Caire and S. Shamai, "On the Achievable Throughput of a Multiantenna Gaussian Broadcast Channel," IEEE Transactions on Information Theory, vol. 49, no. 7, pp. 1691-1706, Jul. 2003

[12] P. Viswanath and D. N. C. Tse, "Sum Capacity of the Vector Gaussian Broadcast Channel and Uplink-Downlink Duality," IEEE Transactions on Information Theory, vol. 49, no. 8, pp. 1912-1921 Aug. 2003.

[13] S. Vishwanath, N. Jindal, and A. Goldsmith, "Duality, Achievable Rates, and Sum-Rate Capacity of Gaussian MIMO Broadcast Channels," IEEE Trans. on Information Theory, vol. 49, no. 10, pp. 2658-2668, Oct. 2003.

[14] W. Yu and J. M. Cioffi, "Sum Capacity of Gaussian Vector Broadcast Channels," IEEE Transactions on Information Theory, vol. 50, no. 9, pp. 1875-1892, Sep. 2004.

[15] W. Yu, W. Rhee, S. Boyd, and J. M. Cioffi, "Iterative Water-Filling for Gaussian Vector Multiple-Access Channels," IEEE Transactions on Information Theory, vol. 50, no. 1, pp. 145-152, Jan. 2004.

[16] N. Jindal, W. Rhee, S. Vishwanath, S. A. Jafar, and A. Goldsmith, "Sum Power Iterative Water-filling for Multi-Antenna Gaussian Broadcast Channels", to appear in IEEE Trans. on Information Theory, 2005.

[17] T. M. Cover and J. A. Thomas, Elements of Information Theory, John Wiley \& Sons, Inc., 1991. 